\title{
Für die Monotherapie ist ein LAMA die erste Wahl
}

Bronchodilatatoren sind das Rückgrat der Therapie einer chronisch obstruktiver Lungenerkrankung (COPD), doch es gibt noch ungeklärte Fragen: Wann kann mit einer Monotherapie begonnen werden und wann ist von Anfang an eine Kombination indiziert? Wenn eine Einzelsubstanz verordnet wird - welches Wirkprinzip sollte dann zum Zuge kommen?

Zur ersten Frage gibt es zwar keine ausreichende wissenschaftliche Evidenz, erklärte Prof. Claus Vogelmeier, Marburg, auf einer Presseveranstaltung in Berlin ${ }^{1}$. Da die COPD aber oft erst in einem Stadium erkannt wird, wenn die Lungenfunktion bereits massiv eingeschränkt ist, werden viele Patienten mit einer Monotherapie nicht auskommen. Vogelmeiers persönliche Strategie ist, eine Einzeltherapie primär bei frisch diagnostizierten, therapienaiven Patienten mit milder Symptomatik zu verordnen. Vorbehandel- te Patienten und solche, die bereits ausgeprägte Symptome zeigen, erhalten gleich eine Kombination.

Entscheidet man sich für die Monotherapie, ist ein lang wirksames Anticholinergikum (LAMA) für Vogelmeier die erste Wahl. Denn inzwischen haben schon mehrere Studien zeigen können, dass LAMA akute Exazerbationen effektiver verhindern als lang wirksame Beta2-Agonisten (LABA). Jüngstes Beispiel ist INVIGORATE mit über 3400 Teilnehmern, die an einer schweren COPD litten ${ }^{2}$ : LAMA und LABA unterschieden sich zwar nicht im Effekt auf die Lungenfunktion, aber der LABA konnte hinsichtlich der jährlichen Exazerbationsrate nicht mit dem LAMA gleichziehen (Die Studie war als NichtUnterlegenheitsstudie angelegt.).

Die Erklärung liegt möglicherweise im Genom: Die Post-hoc-Analyse einer anderen LABA-LAMA-Vergleichsstudie, die zu ähnlichen Ergebnissen gekommen war, ergab, dass Patienten mit einem bestimmten homozygoten Genotyp des Beta2-Rezeptors (R16R) deutlich besser auf den LABA ansprachen als alle anderen. Laut Vogelmeier findet sich dieser Genotyp homozygot nur bei ca. 20\% der Menschen die Mehrheit wird also eher von einem LAMA wie Aclidinium ${ }^{3}$ als Firstline-Therapie profitieren.

Die meisten COPD-Patienten erhalten aber eher zu viel Medikation als zu wenig. Auch Dreifachkombinationen werden verbreitet verordnet, obwohl nie ausreichend untersucht wurde, wer davon profitiert. Insbesondere den breiten Einsatz inhalativer Steroide (ICS) sieht Vogelmeier kritisch, zumal sie das PneumonieRisiko bei COPD steigern. Nach den Leitlinien sind ICS nur indiziert, wenn eine schwere COPD mit häufigen Exazerbationen vorliegt.

\section{Manuela Arand, Berlin}

\footnotetext{
${ }^{1}$ Pneumologisches Kolloquium „PneumoLOQ“, Berlin, Dezember 2014, Veranstalter: Berlin-Chemie AG

${ }^{2}$ Decramer M et al. Lancet Respir Med 2013; 7: 524-533

${ }^{3}$ Bretaris $^{\circledR}$, Berlin-Chemie AG, Berlin
}

\section{Die Schwierigkeit der frühe Diagnose und der Arzt-Patienten-Kommunikation}

Prof. Michael Dreher, Aachen, lenkte bei einer Presseveranstaltung den Blick auf die Lebensspanne, in der Patienten mit chronisch obstruktiver Lungenerkrankung (COPD) an starken krankheitsbedingten Beeinträchtigungen leiden ${ }^{1}$. „COPD steht bezogen auf Disability Adjusted Lifeyears nach ischämischen Herzerkrankungen an 2. Stelle aller krankheitsbedingten Todesursachen“, so Dreher.

Hauptursache der Volkskrankheit ist nach wie vor das Inhalationsrauchen. Eine Schwierigkeit, COPD früh zu erkennen, liegt im zunehmend jüngeren Alter begründet, in dem mit dem Rauchen begonnen wird. In der Konsequenz werden die COPD-Patienten immer jünger. Statt um den klassischen „Pink Puffer“ kann es sich jetzt auch um ca. 40-jährige, gesund wirkende Menschen handeln, wie Dreher erklärte. Ein weiterer Grund für die mangelnde frühzeitige Aufmerksamkeit für eine COPD besteht darin, dass das Hauptsymptom Husten nicht entsprechend bewertet wird. Außerdem spürt der Patient die im weiteren Verlauf auftretende Atemnot zunächst nur bei starker Belastung. Doch die Lungenfunktion nimmt bereits in dem Zeitraum ab, in dem der Patient noch asymptomatisch ist. Die Symptombewertung wird auch durch die Ursachenvielfalt von Husten und Luftnot erschwert. Einen wichtigen Hinweis liefert dabei die Atemfrequenz. „Bei einer Tachypnoe hat der Patient bis zum Beweis des Gegenteils eine Störung des respiratorischen Systems“, sagte Dreher.

\section{Patient muss für Therapie gewonnen werden}

Die Adhärenz bei COPD-Patienten liegt in Deutschland bei unter $40 \%$ und weniger als $5 \%$ der Patienten benutzen ihren Inhaler adäquat. Vor diesem Hintergrund betonte Dr. Henning Kothe, Hamburg, die
Bedeutung der Arzt-Patienten-Kommunikation. „Zu Beginn des Arzt-PatientenKontaktes bleibt dem Arzt nur ein kurzer Moment, den Patienten für sich zu gewinnen bzw. einen Kontakt zu etablieren“, so Kothe.

Wie anspruchsvoll es ist, ein nutzbringendes Arzt-Patienten-Gespräch zu führen, zeigen entsprechende Untersuchungen. Danach stimmen in mehr als der Hälfte der Fälle Arzt und Patient nicht über das hauptsächliche Gesundheitsproblem des Patienten überein. Bei chronisch kranken, entscheidungsfähigen Patienten ist die partizipative Entscheidungsfindung (Shared Decision Making) eine geeignete Form der Arzt-PatientenKommunikation.

\section{Matthias Manych, Berlin}

\footnotetext{
1 Presse-Workshop „Gesichter der COPD - von der Symptomerkennung und -bewertung hin zur optimalen Therapie“, Januar 2015, Berlin, Veranstalter: Novartis Pharma GmbH, Nürnberg
} 